\title{
Endovascular Management of Varicose Veins: A Review of Literature
}

\author{
Soumil Singhal ${ }^{1}$ Mangerira Chinnappa Uthappa ${ }^{1}$ \\ ${ }^{1}$ Department of Intervention Radiology and Intervention Oncology, \\ BGS Gleneagles Global Hospital, Kengeri, Bangalore, Karnataka, \\ India
}

J Clin Interv Radiol ISVIR 2019;3:98-104

\begin{abstract}
Address for correspondence Soumil Singhal, MD, Department of Intervention Radiology and Intervention Oncology, BGS Gleneagles Global Hospital, Kengeri, Bangalore 560060, Karnataka, India (e-mail: drsoumilsinghal75@gmail.com).
\end{abstract}

\begin{abstract}
Keywords

- varicose vein

- intervention

- radiology

Venous insufficiency of the lower limb is a common condition characterized by a spectrum of symptoms, including bulging leg veins, pain, swelling, and ulceration. Various treatment options are available; however, the newer endovascular options are easy, highly effective, safe, and quick in relieving symptoms. Endovascular treatment options include thermal ablation, mechanicochemical ablation, and foam sclerotherapy. This review article briefly describes various scoring systems used in varicose veins, the role of imaging, different management techniques, and guidelines proposed in the management of this condition.
\end{abstract}

\section{Introduction}

A chronic venous disorder is a clinical condition characterized primarily by weakness within the vein wall and associated with valvular dysfunction and venous reflux. ${ }^{1}$ Chronic venous insufficiency (CVI) of the lower limb is a syndrome that includes all the signs and symptoms occurring due to persistent venous hypertension. Patients present with a spectrum of symptoms, including prominent leg veins (telangiectasias, varicosities), heaviness, pain, itching, swelling, muscle cramps, discoloration, and ulceration. ${ }^{2}$

The estimated prevalence of CVI is up to $73 \%$ in female population and up to $56 \%$ in the male population. ${ }^{3}$ The Framingham study showed that $77 \%$ of women older than 70 years and $10 \%$ of women younger than 30 years had varicose veins. ${ }^{4}$ Various risk factors associated with the condition include female sex, prolonged standing/sitting, pregnancy, occupation (e.g., policemen, farmers, teachers), hormonal influence, obesity, family history, and advanced age. ${ }^{5}$ An excellent and thorough understanding of the lower limb venous anatomy and physiology is warranted for an effective treatment. Different treatment options include traditional surgical management and newer endovascular treatments. Endovascular options include thermal ablation, mechanicochemical ablation (MOCA), and foam sclerotherapy.

\section{Pathophysiology}

Venous pressure in the lower limbs is dependent on the proper functioning of the normal ability of the venous system and calf muscles to return blood, the absence of an upstream venous obstruction, and inflow via the arterial system. Failure of these mechanisms can lead to venous hypertension. CVI of the lower limb develops due to several reasons, including valvular dysfunction, venous wall dysfunction, or deep venous hypertension secondary to proximal venous obstruction. Primary valvular insufficiency develops due to structural and intrinsic biochemical changes ${ }^{2}$ in the vein wall, and secondary venous insufficiency develops due to venous thrombosis.

\section{History and Clinical Examination}

A detailed clinical history and physical examination are required for a patient presenting with symptoms of varicose veins. ${ }^{1,6,7}$ Posttreatment follow-up assessing the same clinical parameters helps in evaluating the effectiveness of the procedure, the level of patient satisfaction, and complications associated with the procedure. A clinical questionnaire with essential questions related to how the symptoms of CVI affect the patient's quality of life $(\mathrm{QoL})$ is crucial and is
License terms

() (1) $\Theta \circledast$ 
recommended. Such a questionnaire can be administered just before the consultation. Availability of a questionnaire in languages other than English is highly recommended to increase the yield and effectiveness of the questionnaire. We propose a simplified questionnaire that addresses all the important questions ( - Fig. 1).

Physical examination involves inspection and palpation of the extremity to look for signs of edema, asymmetry, ulceration, and skin manifestations (rashes, hyperpigmentation). Pelvis should be assessed to rule out any underlying pelvic vein insufficiency or iliac vein obstruction. Arterial insufficiency should also be evaluated before deciding any treatment. The findings collected from the patient's history and physical examination should be organized in various standardized disease severity classifications such as the CEAP (clinical, etiologic, anatomical, pathophysiologic) and VCSS (venous clinical severity score) to standardize the clinical findings and treatment outcomes.

\section{Clinical Etiologic Anatomical Pathophysiologic Classification}

The CEAP classification was proposed by the American Venous Forum and endorsed by the Society for Vascular Surgery, which was published first in 1994 and later revised in 2004. ${ }^{8}$ The CEAP classification is a comprehensive assessment system that takes into account the features such as clinical (C) aspects of venous disease, etiology (E) of the venous disease, anatomical (A) location of the disease, and pathophysiologic (P) components of the disease. The condition is clinically (C) classified as (a) C0: no visible or palpable signs of venous disease, (b) C1: telangiectasia or reticular veins, (c) C2: varicose veins, (d) C3: edema, (e) C4a: hyperpigmentation or eczema, (f) C4b: lipodermatosclerosis, (g) C5: healed venous ulcer, (h) C6: active venous ulcer, (i) s: symptomatic, (ache, pain, tightness, skin irritation, heaviness, muscle cramps), and (j) a: asymptomatic. Etiologic (E) classification of the disease includes Ec (congenital), Ep (primary), Es (secondary), and En (no cause identified). Anatomical (A) classification of the venous disease includes As (superficial veins), Ap (perforator vein), Ad (deep veins), and An (no cause identified), and pathophysiologically as Pr (reflux), Po (obstruction), Pr,o (reflux and obstruction), and Pn (no cause identified). ${ }^{8}$ CEAP classification is the most widely used and accepted system to report chronic venous disease. ${ }^{9}$

\section{Venous Clinical Severity Score}

The VCSS is a QoL score used to supplement the CEAP classification. The score allows quantification of disease severity and how it affects the patient's QoL. Ten clinical characteristics are evaluated and graded between scores 0 and 3 to a total score of 30 . The 10 clinical characteristics include (a) pain or discomfort, (b) varicose veins, (c) edema, (d) pigmentation, (e) inflammation, (f) induration, (g) number of active ulcers, (h) active ulcer size, (i) active ulcer duration, and (j) use of compression therapy. It is useful in grading severity of a patient with a CEAP score of C2 and higher, and more so in patients with CEAP class of C4 to C6. The VCSS score reduces both intra- and interobserver variability and allows better assessment and comparison of signs, symptoms before and after the procedure. ${ }^{10,11}$ VCSS minus stocking (VCSS-S) score can be used to assess the effect of mechanical compression on the angiogenesis post varicose treatment. ${ }^{12}$

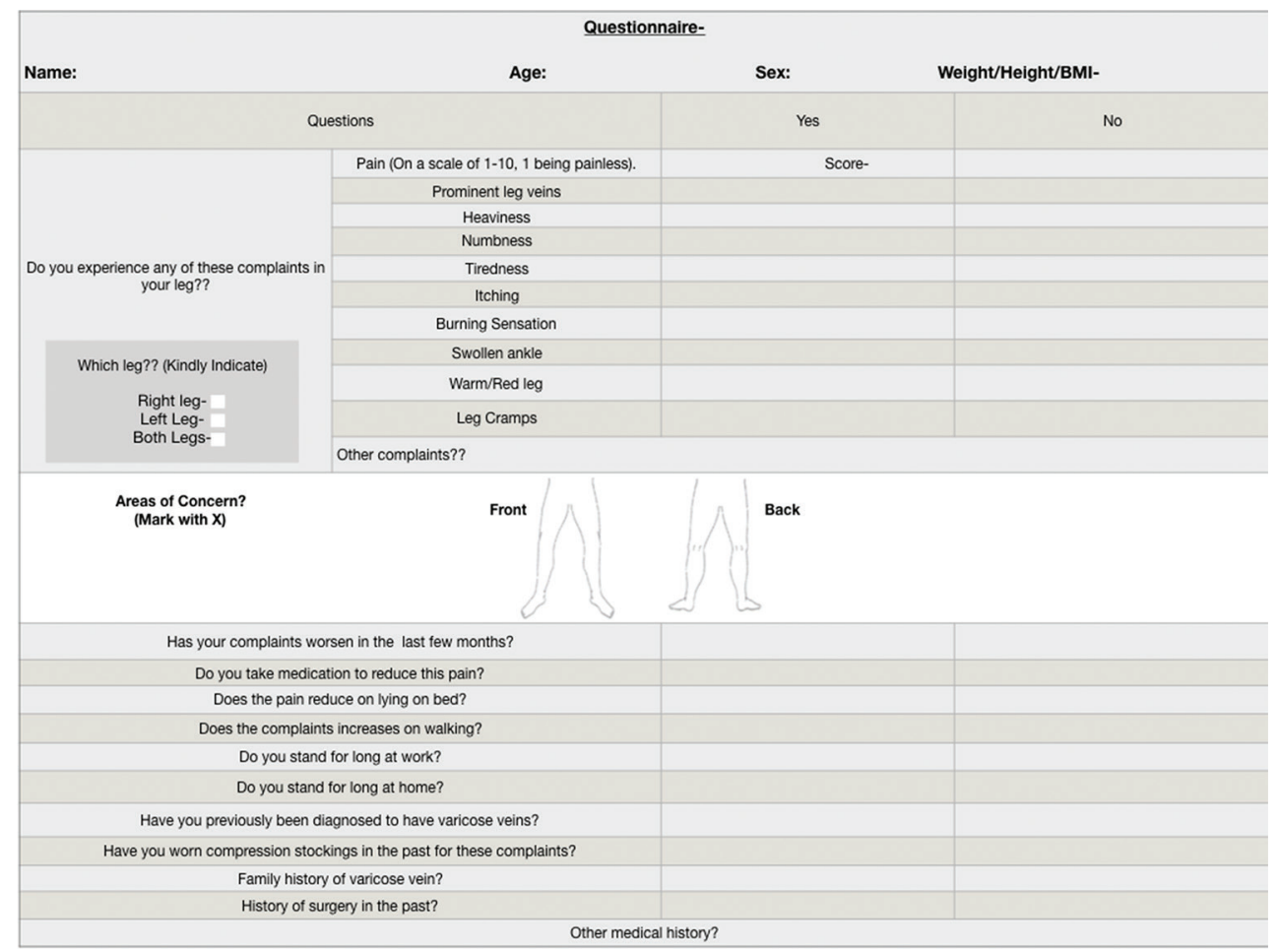

Fig. 1 A simplified proposed pre-consultation questionnaire to assess the patient's condition. 


\section{Quality-of-Life Measure}

Chronic venous disease is associated with depression in up to $30 \%$ of patients. ${ }^{13}$ Assessment of the QoL is essential and integral allowing a thorough and a complete evaluation of the condition. Improvement or deterioration in the disease is associated with a change in the patient's QoL score. Several tools are available to assess QoL; however, they lack sensitivity. The CIVIQ-20 is a 20 -item QoL questionnaire that was created in 1996. ${ }^{14}$ The questionnaire covers four aspects: physical, psychologic, social, and pain. This questionnaire can be accessed on www.civiq-20.com. This questionnaire is disease-specific with high sensitivity and reliability. ${ }^{15}$

\section{Duplex Ultrasound Study}

Duplex scan is the most widely accepted and useful initial imaging tool in the diagnosis of venous insufficiency, its extent, and the treatment planning. The use of both grayscale imaging and pulsed wave Doppler together allows the assessment of both the anatomy and the physiology (concerning the hemodynamics, valvular competency, and venous obstruction) of the lower limb venous system. The addition of color Doppler improves and quickens the ultrasound study. Duplex scan is considered a gold standard for chronic venous disease. The noninvasive nature and the quick reproducibility have allowed the Duplex scan to replace the more invasive procedures such as phlebography that is mainly reserved for exceptional indications. Duplex scan is also a highly useful follow-up tool and helps predict recurrence at saphenofemoral junction (SFJ) at 5 years. ${ }^{16}$

A complete duplex scan incorporates the following features: (a) anatomical information, (b) assessment of flow dynamics, (c) morphology of the valves, and (d) assessment of flow augmentation and venous compressibility ( - Fig. 2).

\section{Scan Protocol}

A standardized duplex evaluation should be performed in a relaxed standing position with the examined leg externally rotated and the weight transferred onto the contralateral

\section{Points to Report on Duplex Scan}

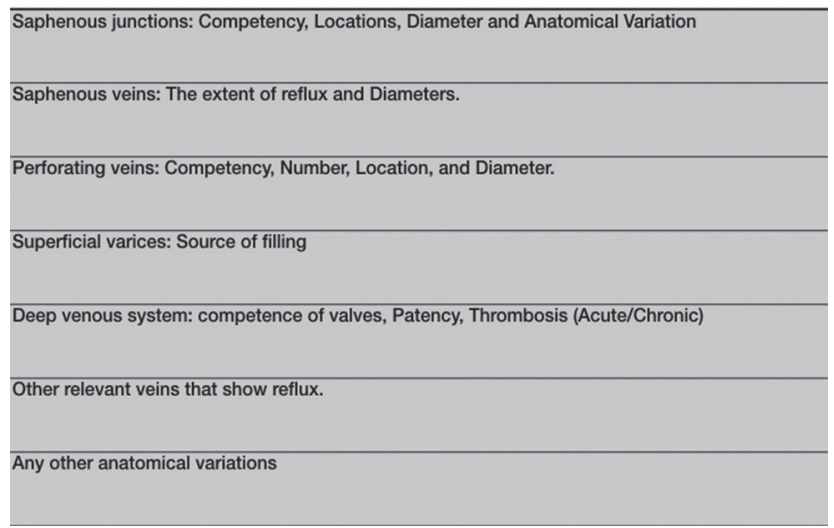

Fig. 2 Points to report for a complete and comprehensive duplex scan. limb. Patency of the iliac vein and common femoral veins should be checked in a supine position, whereas femoral and popliteal vein should be investigated in standing position. Deep veins should be examined with relaxed calf muscles. A high-frequency $(7.5-10 \mathrm{MHz})$ linear array probe with pulse repetition frequency is set to detect low-velocity flow and reflux. The cutoff values to define as reflux in various segments of the venous system include more than 1 seconds in the popliteal vein and femoral vein, more than 0.5 second in the deep femoral vein, superficial venous system, and calf veins, and more than 0.35 second in perforating veins (all of which are in standing position). ${ }^{17}$ The superficial venous system includes the great saphenous vein (GSV), short saphenous vein (SSV), anterior accessory saphenous vein (AASV), and posterior accessory saphenous vein (PASV). Perforator diameter should be measured at the fascial level. The GSV diameter should be taken in three locations: $3 \mathrm{~cm}$ below SFJ, at mid-thigh, and knee. The SSV diameter should be taken $3 \mathrm{~cm}$ below saphenopopliteal junction $(\mathrm{SPJ})^{18}$ (-Fig. 3).

\section{Management}

\section{Conventional Surgery}

Surgical intervention has historically been the treatment of choice for venous insufficiency for more than half a century. Ligation, stripping, and avulsion have been some of the older techniques used. The requirement of hospitalization, general anesthesia, and associated postsurgical complication makes it less attractive for the patient. About 25 to $50 \%$ of patients present with recurrence within 5 years ${ }^{19-21}$ of surgery because of neovascularization, reendothelialization, or incomplete/inadequate/inappropriate treatment.

Newer surgical methods include Cure conservatrice et Hémodynamique de l'Insuffisance Veineuse en Ambulatoire (CHIVA) and ambulatory phlebectomy. Ambulatory phlebectomy is a newer refined surgical technique used by avulsing tributaries under local anesthesia using small stab-like incisions. CHIVA is a shunt ligation technique.

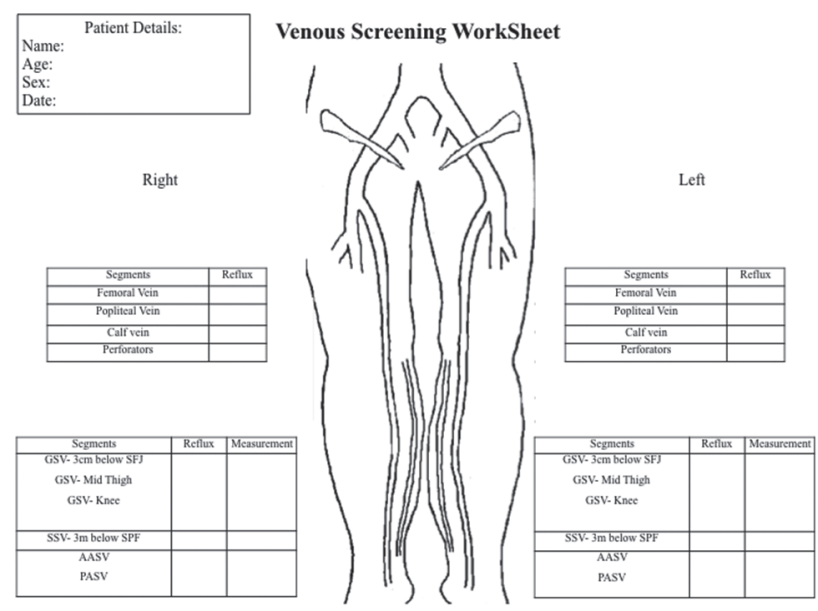

Fig. 3 Venous screening worksheet. 


\section{Compression Therapy}

Compression therapy has been used in the management of ulcers and wounds for several centuries and has undergone the process of evolution from the early days of an inelastic bandage to elastic bandages. Compression therapy remains a crucial component in CVI management because of its noninvasive nature and the ease of its use. Compression therapy counteracts the effect of gravity by decreasing both venous hypertension and interstitial pressure preventing venolymphatic disorder. ${ }^{22}$ It is the Laplace's law that dictates in compressive therapy were external pressure $(\mathrm{P})$ is inversely proportional to the radius of curvature (R) and directly to the tension of the material (T). Various compression devices include graduated compression stockings, bandages, pneumatic compression devices, etc. Graduated compression stockings are the first line of management in venous insufficiency, in patients who cannot undergo an ablative/surgical treatment, or in a postprocedure setup. ${ }^{23,24}$ Some studies suggest that stockings do not provide added benefit over 1 week. ${ }^{25}$ Progressively graduated compression stockings (higher pressure at calf than ankle) are better than degressive graduated compression stockings (higher pressure at the ankle) in terms of improvement in patient symptoms and ease of wearing. ${ }^{26}$ In general, compression stockings are associated with poor patient compliance and skin damage. For an effective treatment, the elastic compression stockings should fit properly and be changed every 2 to 4 months as per the manufacturer's advice.

\section{Sclerotherapy}

Sclerotherapy is a chemical ablation technique performed by injecting chemical irritants into the venous lumen under ultrasound guidance leading to inflammation, thrombosis, occlusion and eventually fibrosis. The chemical irritants, known as sclerosants, can be injected as either liquid or foam. These include sodium tetradecyl sulfate (STS), polidocanol, sodium morrhuate, glycerin, and hypertonic saline. Sclerosant causes irreversible damage by attacking the lipid and cell wall within the endothelium. Sclerosants are commonly used as foam is created by the Tessari technique. Tessari method uses mixing of air and sclerosant using a three-way stopcock. Most commonly used mix is a 4:1 combination of air:sclerosant. ${ }^{27,28}$ Sclerotherapy is indicated in the treatment of truncal incompetence, large varicose veins, reticular vein, telangiectatic veins, and incompetent perforators. Absolute contraindications include allergy to sclerosant, acute deep venous thrombosis, active infection at the site of treatment, or prolonged immobility. Post-sclerotherapy compression stockings are necessary to prevent thrombophlebitis. Sclerotherapy is less time consuming, easily repeatable, relatively painless, and inexpensive with a faster recovery. Studies have reported up to $82 \%$ cosmetic improvement, $90 \%$ symptomatic improvement, and $85 \%$ closure rates. ${ }^{29}$ Complications are seen in up to $1.2 \%$ cases and include drug reactions, pain, venous thrombosis, necrosis, hyperpigmentation, migrainelike headache, transient ischemic attack, visual disturbance, and pulmonary embolism. ${ }^{29}$ Foam sclerotherapy is associated with a $90 \%$ recurrence rate after 6 years, which is a significant problem ${ }^{30}$ requiring repeated sittings. Catheter foam sclerotherapy is a recently introduced technique that is as effective as an ultrasound-guided procedure.

\section{Endovenous Thermal Ablation}

Percutaneous endovenous thermal ablation has emerged as a proven, safe, and effective alternative procedure to conventional surgical stripping. The procedure is associated with several advantages, including minimally invasive nature, outpatient procedure, requirement of only local anesthesia, immediate discharge and ambulation, faster recovery, and less periprocedural morbidity. Two forms of endovenous thermal ablation are commonly used: endovascular laser ablation (EVLA) or radiofrequency ablation (RFA). These techniques mainly require an injection of tumescent liquid around the target vein as a protective cushion for the perivenous tissue by the heat sink effect. The principle of RFA is based on heat that is generated by high-frequency alternating current whereas laser ablation is based on the principle of emission of a monochromatic single wavelength wave (ranging from $<420$ to $10,600 \mathrm{~nm}$ ). The technique for both procedures is same; the procedure is performed percutaneously under ultrasound guidance. The probe is placed 1 to $2 \mathrm{~cm}$ distal to the saphenous junctions, followed by which tumescent fluid made of saline, local anesthesia, sodium bicarbonate, and epinephrine is injected along the full course of the vein. The fiber is withdrawn as the energy is emitted that causes an irreversible intraluminal endothelial damage. Post-procedure compression is recommended. ${ }^{6}$ Laser ablation is associated with an occlusion rate ranging from 77 to $100 \%{ }^{31-33}$ Laser ablation and RFA have almost the same occlusion rates, except the fact that patients treated with RFA have less postoperative bruising and pain. ${ }^{34}$ Postprocedure complications include pain, thrombophlebitis, thromboembolism, skin burns, bruising, hyperpigmentation, paresthesia, and pulmonary embolism. ${ }^{35-37}$

\section{Newer Techniques}

\section{Nonthermal Ablative Techniques}

The success of thermal ablative techniques and medical advances led to the development of various nonthermal ablative techniques that completely obviate the need of tumescent anesthesia, further reducing procedure time and post-procedure pain, bruising, and sensory nerve damage. It mainly includes three techniques: endovenous microfoam sclerotherapy, endovenous MOCA, and cyanoacrylate embolization.

\section{Endovenous Microfoam Sclerotherapy}

Varithena is a preformed polidocanol foam canister that is of pharmaceutical grade and the Food and Drug Administration (FDA) approved for the treatment of incompetent GSV. It is a low-density injectable that contains polidocanol, ultra-low amount of nitrogen, oxygen, and carbon dioxide producing a $1 \%$ microfoam solution. ${ }^{38}$ 


\section{Mechanochemical Endovenous Ablation}

Mechanochemical endovenous ablation (MOCA) is a hybrid endovascular procedure that, as the name suggests, has two components: (1) mechanical abrasion via a special catheter and (2) chemical ablation by injecting foam sclerosant. Sodium tetradecyl sulfate or polidocanol can be used as the sclerosant agent of choice ( - Fig. 4 ). The mechanical damage of the endothelium is caused by the catheters rotating element/sharp tines and the chemical damage by the sclerosants. The mechanically damaged endothelium activates coagulation and causes vasospasm with the sclerosant damaging the lipid cell wall. These together lead to occlusion of the vein. ${ }^{39}$ The procedure is associated with faster recovery and less post-procedure pain and discomfort. ${ }^{40}$ The procedure has several advantages over other ablative techniques, including (a) reduced pain, bruising and discomfort, (b) no tumescent anesthesia, (c) no risk of nerve and skin damage, and (d) rapid return to regular activity. No major complication is associated with this technique. Minor complications such as local site hematoma, thrombophlebitis, and ecchymosis can occur. ${ }^{41}$ Tang et al noted no major complications within their study. Approximately $4 \%$ of the patients presented with thrombophlebitis. ${ }^{42}$ MOCA has shown a closure rate of 87 to $96 \% .^{39,43} \mathrm{~A}$ randomized controlled trial comparing MOCA and RFA showed a closure rate of $92 \%$ at 4 weeks. ${ }^{44}$ Tang et al reported no difference in occlusion while treating GSV and SSV. ${ }^{42}$

\section{Cyanoacrylate Embolization}

The VenaSeal Sapheon Closure System is a proprietary $n$-butyl-2-cyanoacrylate-based formulation. This is injected into the lumen to treat varicose veins. It polymerizes when it comes in contact with blood leading to occlusion of the vessel..$^{45}$

\section{Laser-Assisted Foam Sclerotherapy}

Laser-assisted foam sclerotherapy (LAFOS) is characterized by foam injection that precedes a low-energy laser ablation. No tumescent anesthesia is necessary for this technique. A study showed a $100 \%$ occlusion rate. ${ }^{46}$

\section{Guidelines}

The NICE (National Institute for Health and Care Excellence) guidelines for varicose veins, first issued in 2013, make several recommendations (all recommendations are for patients aged $>18$ years). ${ }^{47}$ Guidelines have also been proposed by a few other vascular societies such as the American Venous Forum, the Society for Vascular Surgery, and the European Society for Vascular Surgery. Most of the available guidelines are in agreement with the guidelines proposed by NICE.

\section{Treatment Comparison}

With the availability of several treatment options choosing the right treatment for the right patient is a crucial decision that needs to be taken. Kheirelseid et al in their meta-analysis noted that $36.6 \%$ patients who underwent EVLA presented with recurrence in comparison with $33.3 \%$ patients who underwent conventional surgery at the end of 5 years. ${ }^{48}$ Xiao et al found no difference in the results of EVLT versus surgery. ${ }^{49}$ One study showed that $50 \%$ of recurrences occurred after 2 years of surgery whereas recurrence was seen in $12 \%$ of patient within 6 months of laser ablation. ${ }^{50}$ Kheirelseid et al found no significant difference in recurrence rate when comparing surgery over RFA. ${ }^{48}$ Luebke et al found that on comparing RFA over surgery, radiofrequency has several short-term benefits; however, there was an increasing rate of recanalization at 1 year. ${ }^{51}$ Studies comparing surgery and endothermal procedure found both to have the same results over the long term; however, the endothermal procedure had several advantages, including it being a safe and effective

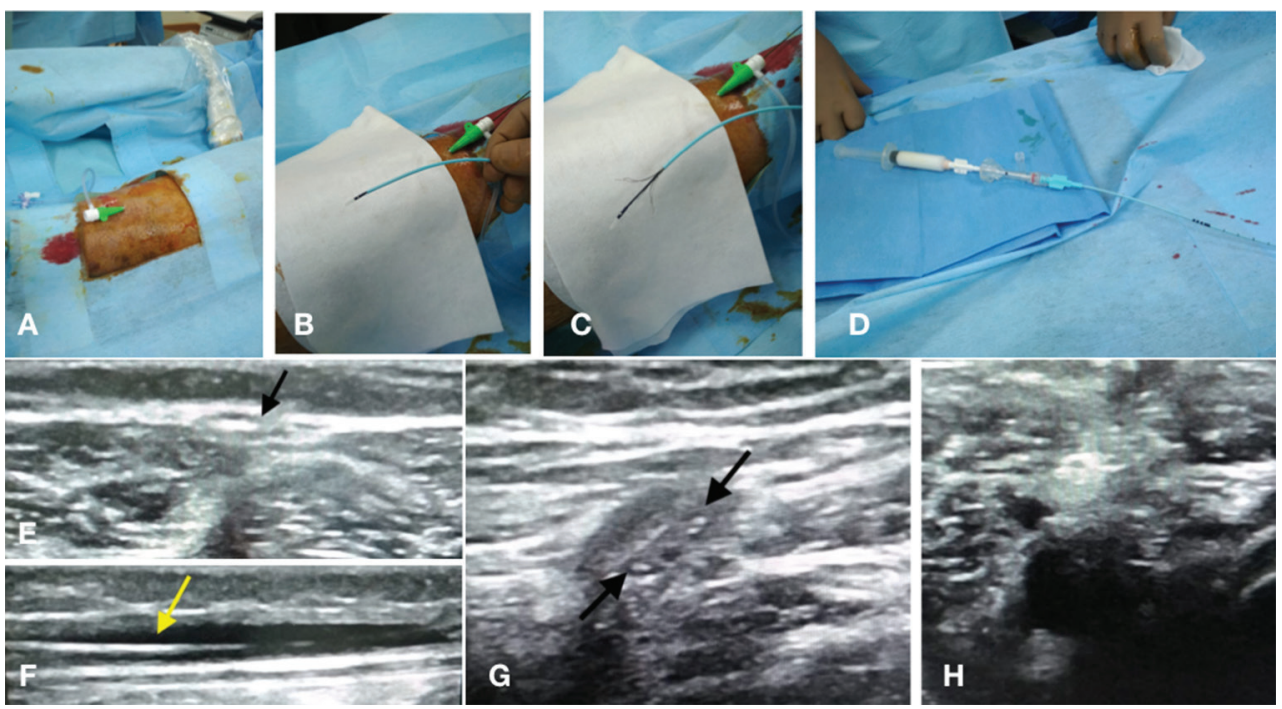

Fig. 4 A 63-year-old male patient first presented with complaints of itching and dull aching pain in the right leg. On screening venous Doppler, there was reflux across the saphenopopliteal junction (SPJ) with a dilated short saphenous vein. Patients CEAP score was C4a and VCSS score was 7. (A-H) Sequential procedure images. (A) Punctured SSV with a 6F sheath within the SSV. (B, C) Flebogrif catheter with opened tines. (D) Foam sclerosant syringe connected to the Flebogrif catheter hub. (E, F) Catheter tip seen in axial section in the SSV (black arrow in E) and in sagittal section (yellow arrow in $\mathbf{F}$ ). (G, H) Catheter tines are opened (black arrows in $\mathbf{G}$ ) with occlusion postmechanical ablation. 
procedure with faster recovery..$^{52}$ RFA and laser ablation had similar outcomes on both short- and long-term follow-up. ${ }^{53}$ Several studies have found minimally invasive procedures as effective and safe, ${ }^{54,55}$ with one study reporting endothermal ablation being superior to surgery. ${ }^{56}$ As suggested by the NICE guidelines, ${ }^{47}$ endothermal treatment is the first line of treatment. In patients not suitable for endothermal ablation, ultrasound-guided foam sclerotherapy must be offered as the next treatment option. Only patients in whom both endothermal treatment and foam sclerotherapy are not a suitable treatment option should surgery be offered.

\section{Conclusion}

Venous insufficiency of the lower limb is a widespread condition that, when diagnosed and treated early, can prevent disease progression and complications associated with the procedure. Principles of ultrasound and intervention are well understood by the interventional radiologist giving them the skills to treat the condition with utmost accuracy ideally. Studies have shown that endovascular treatment offers equal long-term efficacy similar to surgery. Nonthermal endovenous ablations are newer techniques but are in need of longterm outcome data.

\section{Conflict of Interest}

None.

\section{References}

1 Khilnani NM, Grassi CJ, Kundu S, et al; Cardiovascular Interventional Radiological Society of Europe, American College of Phlebology, and Society of Interventional Radiology Standards of Practice Committees. Multi-society consensus quality improvement guidelines for the treatment of lower-extremity superficial venous insufficiency with endovenous thermal ablation from the Society of Interventional Radiology, Cardiovascular Interventional Radiological Society of Europe, American College of Phlebology and Canadian Interventional Radiology Association. J Vasc Interv Radiol 2010;21(1):14-31

2 Meissner MH, Gloviczki P, Bergan J, et al. Primary chronic venous disorders. J Vasc Surg 2007;46 (Suppl S):54S-67S

3 Beebe-Dimmer JL, Pfeifer JR, Engle JS, Schottenfeld D. The epidemiology of chronic venous insufficiency and varicose veins. Ann Epidemiol 2005;15(3):175-184

4 Brand FN, Dannenberg AL, Abbott RD, Kannel WB. The epidemiology of varicose veins: the Framingham study. Am J. Prev Med 1988;4(2):96-101

5 Alexander CJ. The epidemiology of varicose veins. Med J Aust 1972;1(5):215-218

6 Gloviczki P, Comerota AJ, Dalsing MC, et al; Society for Vascular Surgery; American Venous Forum. The care of patients with varicose veins and associated chronic venous diseases: clinical practice guidelines of the Society for Vascular Surgery and the American Venous Forum. J Vasc Surg 2011;53(5 Suppl):2S-48S

7 Kundu S, Grassi CJ, Khilnani NM, et al; Cardiovascular Interventional Radiological Society of Europe, American College of Phlebology, and Society of Interventional Radiology Standards of Practice Committees. Multi-disciplinary quality improvement guidelines for the treatment of lower extremity superficial venous insufficiency with ambulatory phlebectomy from the Society of Interventional Radiology, Cardiovascular Interventional Radiological Society of Europe, American College of
Phlebology and Canadian Interventional Radiology Association. J Vasc Interv Radiol 2010;21(1):1-13

8 Beebe HG, Bergan JJ, Bergqvist D, et al. Classification and grading of chronic venous disease in the lower limbs. A consensus statement. Eur J Vasc Endovasc Surg 1996;12(4):487-491, (discussion) 491-492

9 Rabe E, Pannier F. Clinical, aetiological, anatomical and pathological classification (CEAP): gold standard and limits. Phlebology 2012;27(Suppl 1):114-118

10 Rutherford RB, Padberg FT Jr, Comerota AJ, Kistner RL, Meissner $\mathrm{MH}$, Moneta GL. Venous severity scoring: an adjunct to venous outcome assessment. J Vasc Surg 2000;31(6):1307-1312

11 Vasquez MA, Rabe E, McLafferty RB, et al; American Venous Forum Ad Hoc Outcomes Working Group. Revision of the venous clinical severity score: venous outcomes consensus statement: special communication of the American Venous Forum Ad Hoc Outcomes Working Group. J Vasc Surg 2010;52(5):1387-1396

12 van Rij AM, Jones GT, Hill BG, et al. Mechanical inhibition of angiogenesis at the saphenofemoral junction in the surgical treatment of varicose veins: early results of a blinded randomized controlled trial. Circulation 2008;118(1):66-74

13 Sritharan K, Lane TR, Davies AH. The burden of depression in patients with symptomatic varicose veins. Eur J Vasc Endovasc Surg 2012;43(4):480-484

14 Launois R, Reboul-Marty J, Henry B. Construction and validation of a quality of life questionnaire in chronic lower limb venous insufficiency (CIVIQ) Qual Life Res 1996;5(6):539-554

15 Vasquez MA, Munschauer CE. Venous clinical severity score and quality-of-life assessment tools: application to vein practice. Phlebology 2008;23(6):259-275

16 De Maeseneer MG, Vandenbroeck CP, Hendriks JM, Lauwers PR, Van Schil PE. Accuracy of duplex evaluation one year after varicose vein surgery to predict recurrence at the saphenofemoral junction after five years. Eur J Vasc Endovasc Surg 2005;29(3):308-312

17 Labropoulos N, Tiongson J, Pryor L, et al. Definition of venous reflux in lower-extremity veins. J Vasc Surg 2003;38(4):793-798

18 De Maeseneer M, Pichot O, Cavezzi A, et al; Union Internationale de Phlebologie. Duplex ultrasound investigation of the veins of the lower limbs after treatment for varicose veins-UIP consensus document. Eur J Vasc Endovasc Surg 2011;42(1):89-102

19 Royle JP. Recurrent varicose veins. World J Surg 1986;10(6):944-953

20 Blomgren L, Johansson G, Dahlberg-A, Kerman A, et al. Recurrent varicose veins: incidence, risk factors and groin anatomy. Eur J Vasc Endovasc Surg 2004;27(3):269-274

21 van Rij AM, Jiang P, Solomon C, Christie RA, Hill GB. Recurrence after varicose vein surgery: a prospective long-term clinical study with duplex ultrasound scanning and air plethysmography. J Vasc Surg 2003;38(5):935-943

22 Mariani F, ed. Compression: Consensus Document Based on Scientific Literature and Clinical Experience. Torino, Italy: Edizioni Minerva Medica; 2009

23 Palfreyman SJ, Lochiel R, Michaels JA. A systematic review of compression therapy for venous leg ulcers. Vasc Med 1998;3(4):301-313

24 Lohr JM, Bush RL. Venous disease in women: epidemiology, manifestations, and treatment. J Vasc Surg 2013;57(4 Suppl): 37S-45S

25 Huang TW, Chen SL, Bai $\mathrm{CH}, \mathrm{Wu} \mathrm{CH}$, Tam KW. The optimal duration of compression therapy following varicose vein surgery: a meta-analysis of randomized controlled trials. Eur J Vasc Endovasc Surg 2013;45(4):397-402 
26 Couzan S, Leizorovicz A, Laporte S, et al. A randomized double-blind trial of upward progressive versus degressive compressive stockings in patients with moderate to severe chronic venous insufficiency. J Vasc Surg 2012;56(5):1344-1350

27 Cavezzi A, Tessari L. Foam sclerotherapy techniques: different gases and methods of preparation, catheter versus direct injection. Phlebology 2009;24(6):247-251

28 Tessari L, Cavezzi A, Frullini A. Preliminary experience with a new sclerosing foam in the treatment of varicose veins. Dermatol Surg 2001;27(1):58-60

29 Rathbun S, Norris A, Morrison N, et al. Performance of endovenous foam sclerotherapy in the USA for the treatment of venous disorders: ACP/SVM/AVF/SIR quality improvement guidelines. Phlebology 2014;29(2):76-82

30 Hobbs JT. Surgery and sclerotherapy in the treatment of varicose veins. A random trial. Arch Surg 1974;109(6):793-796

31 Mundy L, Merlin TL, Fitridge RA, Hiller JE. Systematic review of endovenous laser treatment for varicose veins. Br J Surg 2005;92(10):1189-1194

32 Disselhoff BC, der Kinderen DJ, Kelder JC, Moll FL. Randomized clinical trial comparing endovenous laser with cryostripping for great saphenous varicose veins. Br J Surg 2008;95(10):1232-1238

33 Carradice D, Mekako AI, Mazari FA, Samuel N, Hatfield J, Chetter IC. Randomized clinical trial of endovenous laser ablation compared with conventional surgery for great saphenous varicose veins. Br J Surg 2011;98(4):501-510

34 Rasmussen LH, Lawaetz M, Bjoern L, Vennits B, Blemings A, Eklof B. Randomized clinical trial comparing endovenous laser ablation, radiofrequency ablation, foam sclerotherapy and surgical stripping for great saphenous varicose veins. Br J Surg 2011;98(8):1079-1087

35 van den Bos R, Arends L, Kockaert M, Neumann M, Nijsten T. Endovenous therapies of lower extremity varicosities: a meta-analysis. J Vasc Surg 2009;49(1):230-239

36 Gibson KD, Ferris BL, Polissar N, Neradilek B, Pepper D. Endovenous laser treatment of the small [corrected] saphenous vein: efficacy and complications. J Vasc Surg 2007;45(4):795-801, discussion 801-803

37 Lawrence PF, Chandra A, Wu M, et al. Classification of proximal endovenous closure levels and treatment algorithm. J Vasc Surg 2010;52(2):388-393

38 King JT, O’Byrne M, Vasquez M, Wright D; VANISH-1 Investigator Group. Treatment of truncal incompetence and varicose veins with a single administration of a new polidocanol endovenous microfoam preparation improves symptoms and appearance. Eur J Vasc Endovasc Surg 2015;50(6):784-793

39 van Eekeren RR, Hillebrands JL, van der Sloot K, de Vries JP, Zeebregts CJ, Reijnen MM. Histological observations one year after mechanochemical endovenous ablation of the great saphenous vein. J Endovasc Ther 2014;21(3):429-433

40 van Eekeren RR, Boersma D, Konijn V, de Vries JP, Reijnen MM. Postoperative pain and early quality of life after radiofrequency ablation and mechanochemical endovenous ablation of incompetent great saphenous veins. J Vasc Surg 2013;57(2):445-450

41 Sadek M, Kabnick LS. Are non-tumescent ablation procedures ready to take over? Phlebology 2014;29(1 suppl):55-60
42 Tang TY, Kam JW, Gaunt ME. ClariVein ${ }^{\circledR}$-early results from a large single-centre series of mechanochemical endovenous ablation for varicose veins. Phlebology 2017;32(1):6-12

43 Elias S, Lam YL, Wittens CH. Mechanochemical ablation: status and results. Phlebology 2013;28(Suppl 1):10-14

44 Bootun R, Lane TR, Dharmarajah B, et al. Intra-procedural pain score in a randomised controlled trial comparing mechanochemical ablation to radiofrequency ablation: The Multicentre Venefit $^{\mathrm{TM}}$ versus ClariVein ${ }^{\circledR}$ for varicose veins trial. Phlebology 2016;31(1):61-65

45 Morrison N, Gibson K, McEnroe S, et al. Randomized trial comparing cyanoacrylate embolization and radiofrequency ablation for incompetent great saphenous veins (VeClose) J Vasc Surg 2015;61(4):985-994

46 Frullini A, Fortuna B. Laser assisted foam sclerotherapy (LAFOS): a new approach to the treatment of incompetent saphenous veins. Phlebologie 2013;66:51-54

47 National Institute for Health and Care Excellence Varicose veins in the legs pathway http://pathways.nice.org.uk/pathways/ varicose-veins-in-the-legs. Accessed December 30, 2018

48 Kheirelseid EAH, Crowe G, Sehgal R, et al. Systematic review and meta-analysis of randomized controlled trials evaluating long-term outcomes of endovenous management of lower extremity varicose veins. J Vasc Surg Venous Lymphat Disord 2018;6(2):256-270

49 Xiao Y, Chen Z, Yuan C, et al. Comparison of long-term result of endovenous laser ablation vs high ligation and stripping for varicosity of great saphenous vein: a meta analysis. Med J Chin People's Liberation Army 2015;40(9):763-768

50 Liberati A, Altman DG, Tetzlaff J, et al. The PRISMA statement for reporting systematic reviews and meta-analyses of studies that evaluate health care interventions: explanation and elaboration. PLoS Med 2009;6(7):e1000100

51 Luebke T, Gawenda M, Heckenkamp J, Brunkwall J. Meta-analysis of endovenous radiofrequency obliteration of the great saphenous vein in primary varicosis. J Endovasc Ther 2008;15(2):213-223

52 Xenos ES, Bietz G, Minion DJ, et al. Endoluminal thermal ablation versus stripping of the saphenous vein: meta-analysis of recurrence of reflux. Int J Angiol 2009;18(2):75-78

53 Balint R, Farics A, Parti K, et al. Which endovenous ablation method does offer a better long-term technical success in the treatment of the incompetent great saphenous vein? [Review] Vascular 2016;24(6):649-657

54 Nesbitt C, Bedenis R, Bhattacharya V, Stansby G. Endovenous ablation (radiofrequency and laser) and foam sclerotherapy versus open surgery for great saphenous vein varices. Cochrane Database Syst Rev 2014; (7):CD005624

55 Leopardi D, Hoggan BL, Fitridge RA, Woodruff PW, Maddern GJ. Systematic review of treatments for varicose veins. Ann Vasc Surg 2009;23(2):264-276

56 Boersma D, Kornmann VN, van Eekeren RR, et al. Treatment modalities for small saphenous vein insufficiency: systematic review and meta-analysis. J Endovasc Ther 2016;23 (1):199-211 